\title{
UNE STATUE RÉCEMMENT DÉCOUVERTE À VIEUX (CALVADOS)
}

\author{
par Pascal VIPARD*
}

En août 1988, plusieurs fragments d'une statue féminine en calcaire local ont été mis au jour à Aregenua/Vieux (Calvados), dans un bâtiment du deuxième quart du ${ }^{\text {er }} \mathrm{s}$. détruit vers la fin du III $^{\mathrm{e}} \mathrm{s}$. Six éléments seulement subsistent : la tête, la main gauche, la base du vêtement, un élément indéterminé et, surtout, une couronne tourelée et la partie proximale d'une corne d'abondance. Ces deux derniers attributs permettent de reconnaître une divinité protectrice, mais une étude comparative plus poussée sera nécessaire pour déterminer l'identité exacte de la déesse.

In August 1988, several fragments of the statue of a woman, made in a local limestone, were discovered at Aregenua/Vieux (Calvados) in a second quater of the 1st century building, destroyed in the late 3rd century. Only six elements remained : her head and left hand, the lower part of her costume, an unidentified element, and, most of all, a towered crown and the upper part of a cornucopia. The last two elements clearly suggest a protecting deity, but further comparative study will be needed to dertermine her identity.

Située à $10 \mathrm{~km}$ au sud-ouest de Caen, Aregenua, chef-lieu de cité des Viducasses (aujourd'hui village de Vieux, canton d'Evrecy) a fait l'objet d'explorations archéologiques sporadiques depuis le tout début du XviII ${ }^{e}$ s. ${ }^{1}$. En 1988, une première campagne de fouille, menée par le Service départemental d'archéologie du Calvados, a porté sur une partie de la parcelle cadastrale AH 28 sise au lieu-dit «le Bas de Vieux", dans le quartier immédiatement à l'est de deux édifices balnéaires reconnus aux xviII et $\mathrm{xIX}^{e} \mathrm{~s}$. Elle a permis de mettre au jour les vestiges d'un bâtiment luxueux construit dans le deuxième quart du I $^{e r}$ s. et détruit par un incendie vers la fin du III $^{e} \mathbf{s}$. C'est non loin de l'angle nord-ouest d'une pièce

* Service départemental d'archéologie du Calvados, 28, rue Jean-Eudes, 14000 Caen.

1 Consulter notamment M. Besnier, Historique des fouilles de Vieux, Mémoires de la Société Nationale des Antiquaires de France, IX, 1909, p. 225-335, et Chr. Pilet, Vieux antique (Araegenuae, Viducasses), Revue Archéologique de l'Ouest, 1, 1984, p. 63-84. ornée d'une mosaïque, parmi les décombres de la couche de destruction par le feu de l'édifice que les restes d'une statue en calcaire local (pierre de Caen) ont été dégagés. L'exploration du bâtiment et son environnement devant se poursuivre, il nous a paru utile de présenter cette pièce, rare pour la région ${ }^{2}$, dont l'intérêt, tant artistique que religieux, dépasse les limites de la simple histoire locale.

L'œuvre devait se dresser à peu près à l'endroit où elle a été découverte et s'est trouvée renversée et brisée par l'effondrement de la charpente et des murs en torchis lors d'un incendie dont les traces sont nettement visibles sur le calcaire. Seuls six éléments de la statue subsistent : la tête, la couronne, la main gauche, le sommet d'une corne d'abondance, la base du vêtement et un élément indéterminé ; le corps, les

2 A Vieux même, seuls trois fragments de statues ont été mis au jour: en 1697, un corps de Mercure sans tête (Espérandieu 3041) et, en 1705, une tête de femme voilée et une main droite tenant une coupe (Espérandieu 3040). Tous trois sont perdus et n'ont fait l'objet d'aucune reproduction. 

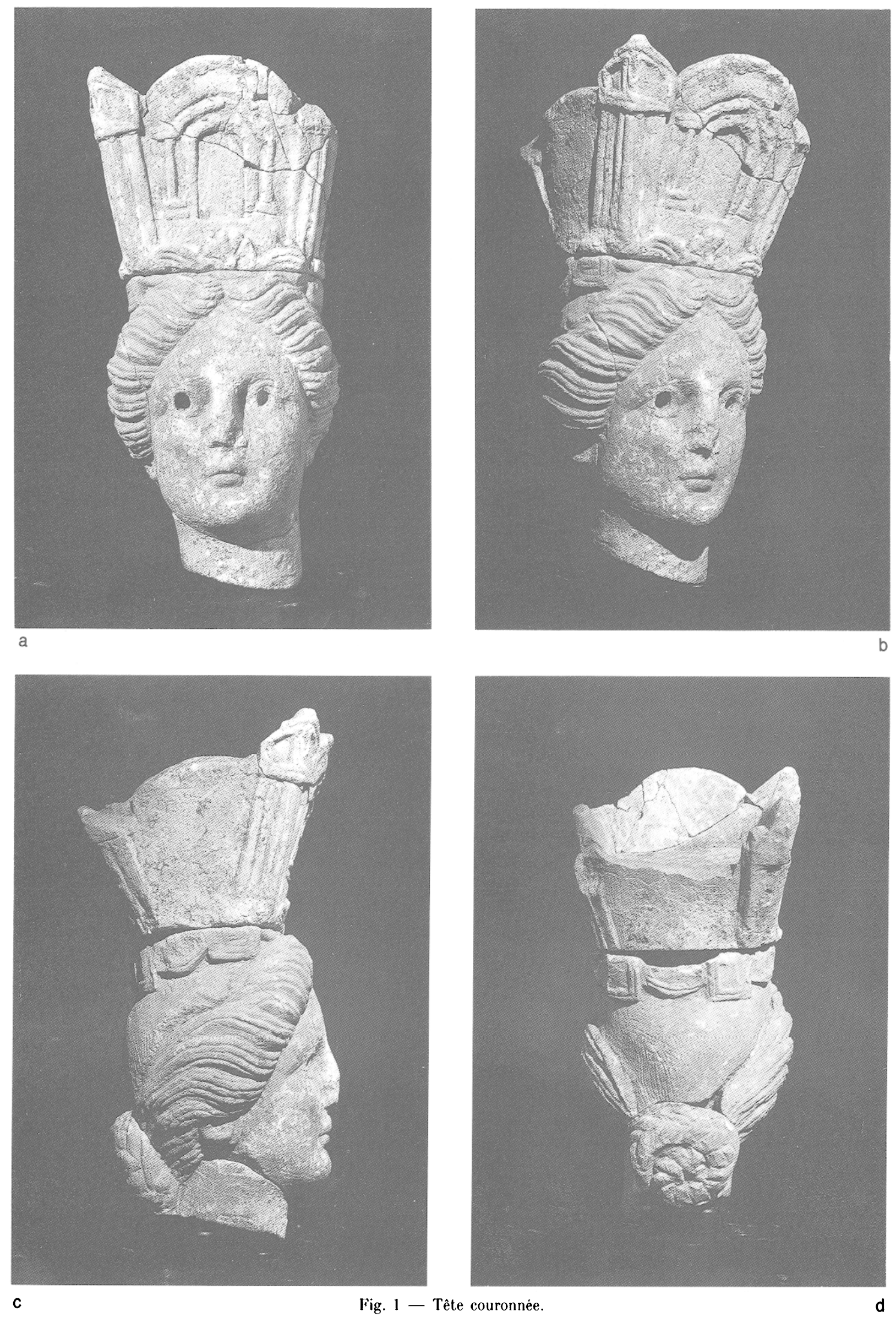

c

Fig. 1 - Tête couronnée

$a$, vue de face; b, vue de trois-quart face; $c$, vue de profil; $d$, vue de l'arrière. 
bras et le socle, non retrouvés, ont probablement fait l'objet d'une récupération après le sinistre ou ont disparu lors du percement d'une voie qui a recoupé postérieurement le bâtiment.

\section{La tête}

Hauteur : $17 \mathrm{~cm}$.

Largeur : $14 \mathrm{~cm}$.

Hauteur du visage : $13,5 \mathrm{~cm}$.

En trois fragments, la tête est celle d'une femme jeune (fig. 1). L'extrémité du nez est brisée, les pupilles sont creuses et étaient remplies de terre lors de la découverte; aucune trace des yeux, même sous forme de pâte de verre fondu, n'a pu être retrouvée. Les cheveux, disposés en deux bandeaux de mèches ondées partant du sommet du front, se rejoignent derrière le crâne pour former un chignon; ils ne laissent découvert que le lobe de l'oreille. Le traitement de la partie postérieure du crâne est assez négligé, l'artiste s'étant contenté de figurer les cheveux tirés par quelques coups de gradine (fig. 1d). Le modèle de ce type de coiffure semble être celui qui avait cours sous les derniers Antonins. C'est celui que l'on trouve représenté sur des monnaies de Faustina II, femme de Marc-Aurèle (RIC III, pl. X et XI ; $B M C, \mathrm{IV}, 2$, pl. 55, 56, 67, $68: 161-176$ après J.-C.), de Lucilla, femme de Lucius Verus $(B M C$, IV, 2 , pl. 77 : vers 164 après J.-C.) ou encore de Crispina, femme de Commode (BMC, IV, 2, pl. 91, $92: 180$ 183 après $\mathrm{J} .-\mathrm{C}$.). On note toutefois chez ces deux dernières un épaississement sensible du chignon qui tendrait donc à rapprocher la coiffure de la déesse de Vieux de celle de Faustina II. L'amorce des tours de la couronne apparaît tout autour du sommet du crâne.

Des traces de polychromie sont encore observables sur le visage, comme sur la plupart des autres fragments : peinture blanc crème sur la face, traces ténues de pigment rouge sur les cheveux ou jaune sur le front.

\section{La couronne tourelée}

Hauteur réelle : $11 \mathrm{~cm}$.

Hauteur en position : 10,5 cm.

Largeur à la base : $11,9 \mathrm{~cm}$.

Épaisseur à la base : $8,5 \mathrm{~cm}$.

Elle s'adapte sur le sommet du crâne (fig. 1). Une légère dépression plus ou moins circulaire sur ce dernier et des traces d'outil étaient destinées à favoriser l'adhésion d'un mortier ou d'une colle quelconque entre la tête et la couronne. Les motifs de la couronne s'efforcent de prendre la suite de ceux de la partie supérieure de la tête, mais si l'on observe une certaine harmonie des tours antérieures, le

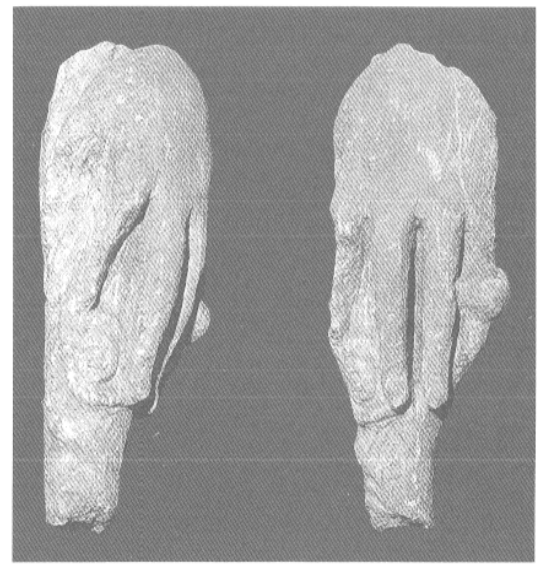

Fig. 2 - Main gauche et base de la corne d'abondance.

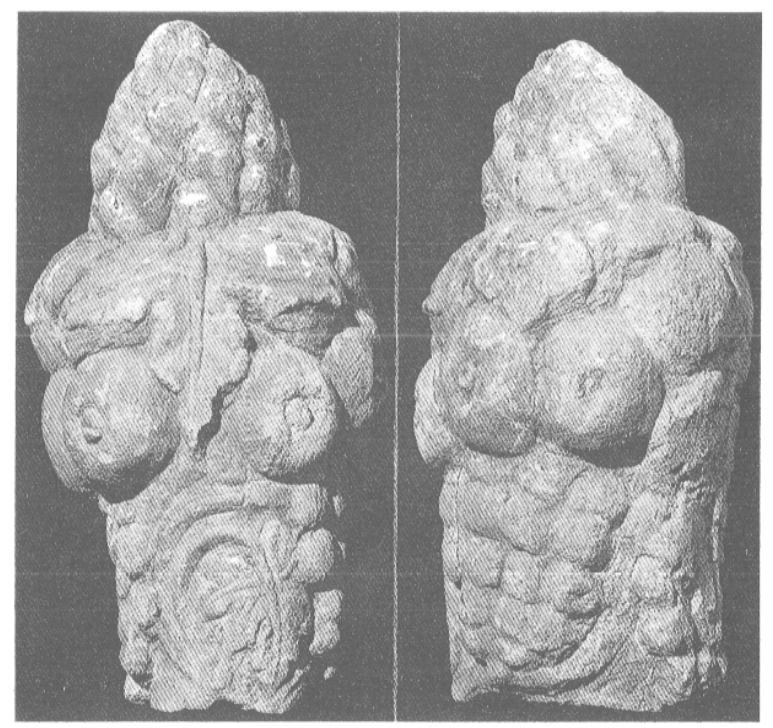

Fig. 3 - Sommet de la corne d'abondance.

sommet des bandeaux de la coiffure et les tours postérieures ne sont pas en parfaite continuité avec leur base; il pourrait s'agir d'une maladresse d'exécution ou d'une réfection légèrement plus tardive.

La face postérieure, avec ses deux tours d'angle, est presque entièrement détruite par le feu; les deux faces latérales, également endommagées par l'incendie, sont vierges de tout décor; seule la face antérieure en présente un : une demi-fleur à six pétales groupés autour d'un cœur circulaire est placée à la base. au milieu et au sommet du front. $\mathrm{Au}$-dessus de ce motif floral, entre les deux tours d'angle, on distingue une voûte cintrée soutenue par deux colonnes avec, légèrement en retrait, une porte. Cet ensemble s'apparente tout à fait à un type de constructions semblables aux arcs triomphaux, mais qui sont, en fait, des portes de monuments ou de 

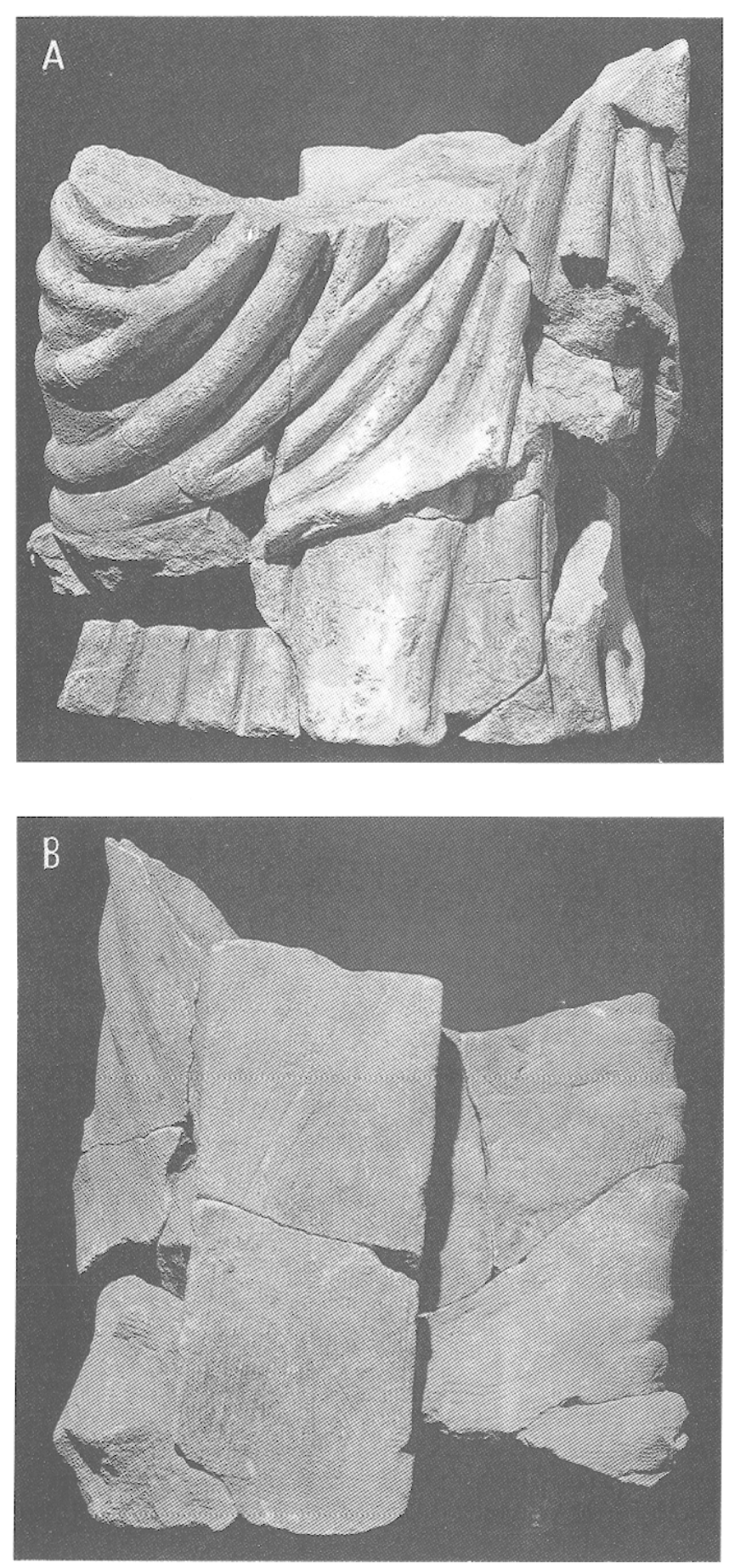

Fig. 4 - Base du vêtement :

$A$, vue de face; $B$, vue de l'arrière.

villes. Dans le cas de la couronne de Vieux, l'existence de tours indique clairement que l'on doit se trouver en présence d'une porte de ville.

\section{La main gauche}

Longueur totale : $14 \mathrm{~cm}$.

Longueur de la main : $10,5 \mathrm{~cm}$.

Largeur : $5,5 \mathrm{~cm}$.

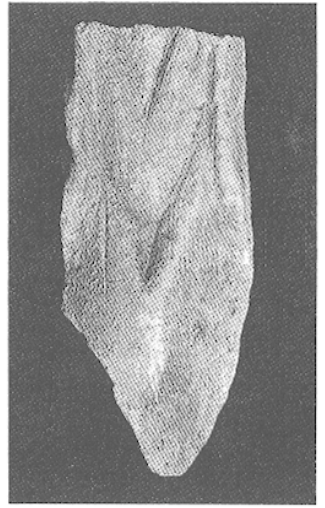

Fig. 5 - Elément indéterminé.

Brisée à la base du poignet, elle présente des doigls très effilés (le pouce et l'index sont en partie détruits) munis d'ongles bien dessinés; une grosse bague orne l'auriculaire (fig. 2).

Elle tient la partie inférieure d'une corne d'abondance de section piriforme, ornée de spirales sinistrogyres et dextrogyres.

\section{La corne d'abondance}

Hauteur : $20 \mathrm{~cm}$.

Largeur : $10,5 \mathrm{~cm}$.

Circonférence maximale : $34,5 \mathrm{~cm}$.

Toute la partie supérieure de la corne d'abondance (fig. 3) est conservée. On y reconnaît, de bas en haut : deux grappes de raisin et une feuille de vigne surmontée de six pommes, elles-mêmes dominées par trois feuilles de figuier; le sommet est constitué d'une pomme de pin. Comme dans la partie inférieure, la section de la corne est piriforme. La réalisation des feuilles et des fruits situés sur la partie originellement tournée vers l'arrière et reposant sur le bras (et peut-être sur l'épaule) est beaucoup moins soignée.

\section{La base du vêtement}

Hauteur : $24 \mathrm{~cm}$.

Largeur : $25 \mathrm{~cm}$.

Epaisseur : $16 \mathrm{~cm}$.

Onze fragments épars ont permis de reconstituer la base du vêtement de la divinité. Il semble s'agir de la partie inférieure d'une tunique longue plissée couvrant entièrement les pieds, sur laquelle vient s'ajuster le bas d'un manteau (fig. 4 a). Un bandeau vertical non décoré (traces de gradine) existe sur toute la partie postérieure conservée (fig. $4 \mathrm{~b}$ ). Une mortaise $(6 \mathrm{~cm} \times 4 \mathrm{~cm} \times 9 \mathrm{~cm})$ située sous le pied atteste l'existence d'une fixation sur un socle. 
Élément indéterminé

Longueur : $13,2 \mathrm{~cm}$.

Largeur : $8,5 \mathrm{~cm}$.

Epaisseur : $3,8 \mathrm{~cm}$ à $4 \mathrm{~cm}$.

Cet élément, se terminant par une pointe, a également été recueilli (fig. 5). Il présente une face postérieure plate (traces de gradine) et une face antérieure semblant être un fragment de drapé.

Malgré l'absence de plus de la moitié de l'œuvre originelle, les divers éléments conservés permettent d'identifier une statue de divinité féminine haute d'environ $1 \mathrm{~m}$ à $1,10 \mathrm{~m}$, couronne comprise. La double présence d'une corne d'abondance et d'une couronne tourelée indique qu'il s'agit d'une déesse de type Forluna ou divinité apparentée ${ }^{3}$. Le caractère urbain nettement affirmé de la couronne ${ }^{4}$ orienterait

3 Voir les remarques de St. BocCHER, Recherches sur les bronzes figurés de la Gaule pré-romaine et romaine, Bibliothèque des Écoles Françaises d'Athènes et de Rome, 228, 1976, p. 148.

4 Les couronnes les plus voisines, mais non identiques, s'observent sur les Tutèles de Mâcon et de la vallée du Rhône alors plutôt l'identification vers une Tutela qui représente un aspect particulier de Fortuna ou du Genius Loci, génie dont le culte a surtout eu pour fidèles non seulement les cités elles-mêmes, mais aussi des magistrats municipaux ou de simples particuliers ${ }^{5}$. Une identification avec Cybèle dont la présence est moins bien attestée en Gaule, mais qui, à titre de divinité protectrice des villes, partage quelquefois les mêmes attributs, pourrait également être retenue.

\section{Pascal Vipard}

(St. Boucher, op. cit., p. 151-152 et nos 264, 267, 268), sur le Génie de Detzem (J.-F. Braemer, L'Art de l'Occident romain, Paris, 1963, p. 103, no 454 ; sans porte cependant) et surtout sur le Genius Loci de Niederbieber (Espérandieu, Complément du Recueil général des bas-reliefs. Stalues et bustes de la Gaule romaine, $n^{\circ}$ 1, p. 2-3) dont l'identité est attestée par une inscription.

5 J. Toutaln, Les cultes païns dans l'Empire romain, I, 1907 , p. $448-456$.

N.B. - Tous les clichés sont de Gilles Lecrosnier (Centre régional de documentation pédagogique de Caen). 